\title{
Inter-Professional Collaborative Care: A Way to Enhance Services for Adults with Intellectual Disability and/or Autism Spectrum Disorder and Mental Health Problems
}

\author{
Jane Summers ${ }^{1,2^{*}}$, Christina Bartha ${ }^{1,2}$, Pushpal Desarkar ${ }^{1,2}$, Lisa Duggan ${ }^{3}$, \\ Julia Fineczko ${ }^{1,4}$, Lew Golding ${ }^{1}$, Ali Shahrami ${ }^{1}$ and Christopher Uranis ${ }^{1,4}$ \\ ${ }^{1}$ Underserved Populations Program, Centre for Addiction and Mental Health, Toronto, Ontario, Canada \\ ${ }^{2}$ Department of Psychiatry, University of Toronto, Toronto, Ontario, Canada \\ ${ }^{3}$ Organizational Development, Centre for Addiction and Mental Health, Toronto, Ontario, Canada \\ ${ }^{4}$ Bloomberg Faculty of Nursing, University of Toronto, Toronto, Ontario, Canada
}

\begin{abstract}
This article describes our inter-professional mental health service for adults with intellectual disability and/or autism spectrum disorder. The service consists of an inpatient unit and outpatient program that are closely aligned and operate within a mental health and addictions teaching hospital. We provide information about recent changes to our model of care and the structures and activities that are used to support inter-professional team development and team functioning. Roles and functions of different mental health professionals on the team are outlined and case examples of adults with intellectual disability and complex mental health needs are provided to illustrate how the inter-professional team members work together.
\end{abstract}

Keywords: Inter-professional mental health team, intellectual disability, autism spectrum disorder, collaborative care, inpatient and outpatient services.

\section{INTRODUCTION}

Individuals with intellectual disability are a complex and vulnerable group. They experience high rates of psychiatric disorders, emotional disturbances and/or behavior problems which are related to a combination of biological, psychosocial and environmental factors [1]. Assessment and intervention for mental health problems may be challenging in this population, particularly in the case of individuals with severe cognitive and communication impairments [2]. One potential way to address these challenges is through the provision of mental health services by specialist teams that are composed of multiple disciplines [3]. These teams should have working relationships with primary care and mainstream mental health services while actively collaborating and partnering with families and care providers as well as the individual with an intellectual disability [4].

This article outlines our newly redesigned program for adults with intellectual disability and/or autism spectrum disorder who also have mental health problems or severely challenging behavior. The Adult Neurodevelopmental Service consists of an outpatient program and brief intervention inpatient unit that are

*Address correspondence to this author at the Underserved Populations Program, Centre for Addiction and Mental Health, 80 Workman Way, Room 5232, Toronto, ON, M6J 1H4, Canada; Tel: 416 535-8501 ext. 30833;

E-mail: jane.summers@camh.ca closely aligned and operate within a large mental health and addictions teaching hospital in Toronto, Canada. We describe recent changes to our model of care and provide background information about these changes, including the inter-professional competency framework that guides our efforts. We provide information about activities to assess and strengthen team functioning. We outline the roles of the different mental health professionals on our team and use case examples of adults with intellectual disability and complex mental health needs to illustrate how our interprofessional team members work together.

\section{HISTORY OF THE ADULT NEURODEVELOP- MENTAL SERVICE}

The new Adult Neurodevelopmental Service started out as a 30-bed provincial psychiatric inpatient unit for adults with intellectual disability at the Queen Street Mental Health Centre in Toronto. In the mid-to-late 1990s, large-scale efforts were underway across Ontario to deinstitutionalize individuals with intellectual disability [5]. Against this backdrop, the focus of the inpatient unit shifted from a chronic psychiatric population to a smaller unit for individuals with a "dual diagnosis" (developmental disability and mental health problem). Over a 5-year period, many long-time clients were discharged to the community and the Dual Diagnosis Program began to accept new admissions in 
1998. The program expanded shortly afterward to include outpatient assessment and consultation.

The Dual Diagnosis Program underwent further change following the merger of four organizations to form the Centre for Addiction and Mental Health (CAMH) in 1998. A new clinical infrastructure was created at $\mathrm{CAMH}$ and strategic priorities for the hospital were established, with increased emphasis being placed on training, education and research. Over the next few years, the structure of the Dual Diagnosis Program remained unchanged while a number of challenges arose. One major obstacle the program faced was discharging clients from hospital beds due to a shortage of supportive housing in the community. As a result, the lengths of hospital stay stretched into years. The number of clients with autism spectrum disorder (ASD) presenting to the hospital increased, reflecting the growing numbers of individuals being diagnosed with ASD and the lack of services and supports to meet their needs. A final challenge was in relation to the demand for specialized assessment and treatment for clients with severely challenging behavior, many of whom had lacked communication skills and had a range of co-morbid medical conditions. In the inpatient unit, high rates of staff and co-client injury and low team morale created a difficult environment for clients, staff and leadership of the program.

\section{EXTERNAL REVIEW AND PROGRAM REDESIGN}

In 2012, CAMH underwent a major program restructuring. Formerly a small stand-alone program, the Dual Diagnosis Program was integrated into the much larger Underserved Populations Program (consisting of Geriatrics, Dual Diagnosis and Child, Youth and Family). An extensive external review of the Dual Diagnosis Program was subsequently carried out. One of the key findings from the review was the need for changes to inpatient and outpatient interprofessional staffing. More specifically, a greater capacity to provide consistent behavioral interventions (across all inpatient shifts in particular) to enhance quality of care and improve safety was identified as a gap across the inpatient and outpatient teams. Specialized leadership and inter-professional education in emerging and best practices were also seen as essential. A greater emphasis was recommended on outpatient care and cross-sectoral collaboration and partnerships with primary care and community agencies serving individuals with intellectual disability. The need to support staff during this period of change was recognized and a commitment was undertaken to strengthen the leadership of the program and promote inter-professional team development. For this reason, we reviewed, redesigned and established a new service model called the "Adult Neurodevelopmental Service." A major component of the model was the enhanced emphasis on behavior therapy and the recruitment of developmental service workers who were formerly not part of the team membership.

\section{DESCRIPTION OF THE OF ADULT NEURODEVE- LOPMENTAL SERVICE}

The Adult Neurodevelopmental Service, which is different from its predecessor the Dual Diagnosis Program, is organized into redesigned clinics/care pathways. The "front door" for most referrals to the service is through a centralized access point for the hospital. After initial processing, referrals are routed to the Adult Neurodevelopmental outpatient program and an intake assessment is carried out. Information generated by the assessment is used to direct the client to one of the following clinics or care pathways: 1) psychiatric consultation clinic; 2) medication consultation clinic; 3) high functioning autism pathway; 4) inter-professional assessment and treatment pathway; or 5) intensive intervention pathway. The brief intervention inpatient unit (BIU) pathway may be accessed through one of the outpatient clinics/care pathways, through the hospital's emergency department or another inpatient unit in the hospital. Once a client is discharged from the BIU, follow-up services are provided through one of the outpatient clinics/care pathways. Staff from the BIU also provide consultation regarding the mental health needs of clients with intellectual disability to other programs within the hospital.

\section{INTER-PROFESSIONAL COLLABORATION}

Individuals with intellectual disability and mental health problems often present with a range of needs that span multiple providers and service systems [6]. An effective response to this situation requires interprofessional collaboration, which makes use of the expertise of different professionals who work together towards a shared goal of providing high quality client care [7]. Inter-professional collaboration aims to establish a partnership between health care providers and the client (including family members or other caregivers) which is a participatory and interactive relationship and provides a coordinated approach to care. Decision making is not left solely to the discretion of one care provider but involves all relevant team 
members as well as the client in a shared decisionmaking process. There are a number of benefits of inter-professional collaboration. For clients, these benefits may include better health outcomes and increased access to health care; for health care staff, greater job satisfaction and less stress and burn out; for a health care organization, greater staff efficiency and more effective use of resources $[8,9]$.

The process of inter-professional collaboration involves an examination of the competencies, skill sets and knowledge base that each health care professional brings to the team with the goal of optimizing and elevating the health outcomes and quality of care received by clients, their families and caregivers. Additionally, each profession's contributions to care and decision making will be modified, enhanced or changed based on input from other members of the team. Inter-professional collaborative approaches vary depending on the complexity of the client population. In the case of individuals with intellectual disability and mental health problems, multiple disciplines working together play a critical role in advancing the required care and goals of the clients and their families and caregivers [10].

The leadership of the Adult Neurodevelopmental Service recognized the need for appropriate organizational structures and supports to be in place in order for team members to develop and optimize the competencies that are required for inter-professional collaborative care.

These competencies include effective communication and group function, role clarification and optimization, collaborative leadership and decision making, conflict resolution, professional and team development $[11,12]$. Given the new disciplines and staff who joined the service, an overarching change management strategy included an intentional focus on inter-professional collaboration.

\section{SURVEY OF TEAM COLLABORATION}

The current state of inter-professional collaboration on the Adult Neurodevelopmental Service was assessed by surveying the leadership of the team as well as the individual team members. A number of positive findings emerged from the leadership survey. Team members were viewed as treating each other with respect; asking each other for help and offering assistance when needed; giving credit to others for their contributions; and sharing information. A number of areas for improvement around inter-professional team collaboration were identified as well, including the need for team members to interact with each other on a more regular basis; talk openly about their feelings and listen attentively to opinions of others; and express clarity about group goals.

As part of a program-wide staff survey, team members from the Adult Neurodevelopmental Service were asked to complete an anonymous survey to indicate their current degree of confidence regarding their inter-professional knowledge and skills. In general, staff viewed themselves as having the knowledge or skills to accurately define what is meant by collaborative practice and the essential elements for effective collaboration; to describe their professional role and competencies to other disciplines; and to recognize and respect the separate and shared roles and competencies of other disciplines. Some issues were identified in regard to working with other disciplines to resolve conflict; to communicate and behave in a way to ensure members feel respected and understood; and negotiate shared roles and responsibilities with their teammates. Thematically, some of the most frequent barriers to better interprofessional collaboration consisted of inadequate time to engage in inter-professional discussion and team building; lack of clear and timely communication; and role confusion.

\section{STRUCTURAL SUPPORTS AND TEAM ACTIVITIES TO PROMOTE AND STRENGTHEN INTER- PROFESSIONAL COLLABORATION}

The Adult Neurodevelopmental Service is led by point-of-care teams for inpatient and outpatient pathways that consist of the manager, psychiatrist and advanced practice clinician. These point-of-care teams are responsible for daily clinical operations while an executive leadership team provides input and support regarding strategy and planning. At each level, the leadership teams work closely together to make joint decisions, but at point of care, this model is particularly important in addressing operational and clinical issues in a coordinated manner, speaking with "one voice" to promote clear and consistent communication with staff. An important activity for each leadership team has been to clarify roles and accountabilities through a discussion of "yours, mine and ours." This approach has since been extended to clinical staff who have worked together to build discipline-specific role clarity charts.

Leadership teams drafted "road maps" to guide and support members of the Adult Neurodevelopmental Service through changes to the model of care and 
foster a revitalized culture of inter-professionalism. Road maps were organized around the common goal of providing high quality, evidence-based services that utilize the expertise of different mental health professionals who work together and alongside the individual and his or her caregivers. Working groups made up of different disciplines helped to operationalize care pathways and advise leadership on issues impacting workflow and team processes, increasing "buy in" and ownership for outcomes. Staff from organizational development met with members of the inter-professional teams to lead them through the process of developing a team charter. The team mission, goals and guidelines for working together were outlined as part of this charter and a commitment from all members was sought. Wood et al. [13], in studying the implementation of care pathways in an intellectual disability service, concluded that leadership plays an important role in guiding and supporting service delivery and managing staff reactions to change.

An important component of inter-professional collaboration relates to a joint appreciation for the training, competencies, roles and responsibilities held by different professions on the team. In fact, the development of mutual respect and understanding among team members may be viewed as an element of "enabling group conditions" that enhance team effectiveness [14]. As part of a team-building exercise, discipline-specific working groups were asked to prepare presentations about their educational background, professional competencies, standards and scope of practice, particularly as they related to working with individuals with intellectual disability and mental health/behavior problems. Inpatient and outpatient team members came together for a collaborative half-day educational event when each working group presented the material they had prepared, followed by a question and answer period. Prior to the presentations, organizational development set the stage for the session by discussing interprofessional collaboration and team building. Presentations were compiled into a comprehensive document and made available to team members as a reference guide.

On an ongoing basis, opportunities for team members to learn about, from and with each other are built into regular team meetings. Members of the interprofessional team take turns presenting on a range of topics that are related to professional development and clinical care. Clinical team meetings and case conferences provide a forum for team discussion and shared decision making about clinical care. Administrative meetings are used to discuss issues related to quality and safety, workflow and team processes.

\section{ROLES AND FUNCTIONS OF INTER- PROFESSIONAL TEAM MEMBERS}

The roles and functions of the inter-professional team members are listed below. Our plans include adding a nurse practitioner to the team to conduct more extensive health assessments and assist with monitoring medications.

\begin{tabular}{|c|c|}
\hline Discipline & Role and Function \\
\hline $\begin{array}{l}\text { Behavior } \\
\text { Therapist }\end{array}$ & $\begin{array}{l}\text {-conduct assessment of functional behavior and } \\
\text { communication, social and environmental issues } \\
\text {-establish target behaviors for intervention plan, } \\
\text { develop observation and monitoring system } \\
\text {-develop safety and proactive support plans, skill } \\
\text { building programs } \\
\text {-train caregivers to implement care plans }\end{array}$ \\
\hline $\begin{array}{l}\text { Developmental } \\
\text { Services } \\
\text { Worker }\end{array}$ & $\begin{array}{l}\text {-deliver individual and group therapeutic } \\
\text { programming } \\
\text {-support observational data collection, } \\
\text { implementation of behavior plans and activities of } \\
\text { daily living }\end{array}$ \\
\hline $\begin{array}{l}\text { Occupational } \\
\text { Therapist }\end{array}$ & $\begin{array}{l}\text {-conduct functional, safety and risk, sensory, } \\
\text { environmental, leisure and vocational assessments } \\
\text {-address areas of self-care, productivity and leisure } \\
\text { pursuits } \\
\text {-develop sensory protocols and protocols for the } \\
\text { use of adaptive equipment }\end{array}$ \\
\hline $\begin{array}{l}\text { Psychiatric } \\
\text { Nurse }\end{array}$ & $\begin{array}{l}\text {-review medical and mental health history, conduct } \\
\text { risk and pain assessments, provide clinical } \\
\text { impressions, educate client and caregivers about } \\
\text { mental health conditions, medications and side } \\
\text { effects }\end{array}$ \\
\hline Psychiatrist & $\begin{array}{l}\text {-conduct mental health assessment and provide } \\
\text { psychiatric diagnosis } \\
\text {-review medical and medication history } \\
\text {-provide biopsychosocial formulation } \\
\text {-liaise with general practitioner } \\
\text {-obtain informed consent for medication } \\
\text {-request specialist input (e.g., neurology, genetics, } \\
\text { internal medicine) } \\
\text {-provide clinical leadership to the inter-professional } \\
\text { team }\end{array}$ \\
\hline Psychologist & $\begin{array}{l}\text {-conduct assessment of cognitive, nonverbal, } \\
\text { adaptive, language, and social-emotional } \\
\text { functioning } \\
\text {-provide biopsychosocial formulation } \\
\text {-co-lead CBT groups for high functioning } \\
\text { individuals with ASD }\end{array}$ \\
\hline Social Worker & $\begin{array}{l}\text {-conduct assessment of family systems, caregiver } \\
\text { stress, service needs } \\
\text {-provide case management and individual, family } \\
\text { and group psychoeducation } \\
\text {-co-lead CBT and caregiver support groups }\end{array}$ \\
\hline $\begin{array}{l}\text { Recreational } \\
\text { Therapist } \\
\text { (inpatient only) }\end{array}$ & $\begin{array}{l}\text {-develop and implement individualized or group } \\
\text { recreational therapeutic programs } \\
\text {-provide therapy to improve social interaction skills, } \\
\text { leisure activity skills }\end{array}$ \\
\hline
\end{tabular}


CASE EXAMPLES TO ILLUSTRATE HOW INTERPROFESSIONAL TEAM MEMBERS WORK TOGETHER

\section{Scenario 1: Sam}

"Sam" is a 45-year-old man who has a diagnosis of autism spectrum disorder and severe intellectual disability. His speech is primarily limited to single words but he may use up to 3-word phrases to express his wants and needs. He also has a seizure disorder and drinks water excessively. Sam was transferred to the Adult Neurodevelopmental inpatient program 6 months ago from an acute care hospital unit where he was kept in arm restraints to prevent him from engaging in severe self-injurious behavior (biting his arms and hands) and to permit his wounds to heal. Prior to his admission to our unit, Sam had been in and out of hospital numerous times in the past 18 months, primarily for treatment of wounds caused by selfinjurious behavior (SIB).

Sam started to develop head-banging at age 2. The behavior tended to occur when he was anxious, when there was a lot of noise or when there were changes in his environment. Arm biting was reported to have developed over a decade ago. Sam was seen in the emergency department 10 years ago because of hand biting. Up until five years ago, Sam's SIB occurred less than once a week and at a low intensity. The SIB caused superficial marks on his hands but no broken skin. Staff at Sam's group home noticed he would bite the lids off plastic bottles as well as chew on metal keys and chew through wires. He also engaged in selfstimulatory behavior (holding a plastic bottle in his hands and flicking it). In addition to the self-biting, Sam had a history of head-banging against hard objects if asked to wait and head butting staff if they were in the way or prevented him from doing tasks such as collecting the garbage. The proposed function of Sam's SIB was to gain access to preferred items (e.g., water to drink, garbage to put away) or escape from unpleasant situations (noisy or crowded conditions).

Sam's self-biting increased in the spring of 2014, to the point where he required hospitalization to manage and treat extremely complex wounds to his arms due to severe biting. His behavior was worsening - increasing frequency of biting and at a greater depth. The biting led to a cycle of significant lacerations and inflammation and affected his muscle and tendons. Quite often the bite was so intense that the bone was exposed. No clear triggers were identified but reportedly the biting got worse after group home staff prevented Sam from chewing metals and plastic objects. His more recent hospitalizations (prior to the Adult Neurodevelopmental inpatient unit) were for treatment of macerated skin from his wrist to elbow. Sam needed plastic surgery and wound care was arranged. The group home staff had tried gloves and considered using a helmet with a face shield.

\section{Assessment and Treatment}

Sam's inter-professional clinical team consisted of the psychiatrist, advanced practice clinician (psychologist), nurses, behavior therapist, developmental service workers, occupational therapist, and social worker. The team worked closely with Sam's substitute decision maker and staff from his group home in the community. The main goal of Sam's interprofessional plan of care was to eliminate his SIB (arm and hand biting) using least intrusive and least restrictive strategies which could be implemented in the community and at his residence.

In order to identify factors that may have been contributing to Sam's SIB, a number of assessments were conducted. A medication review by the psychiatrist indicated that Sam's multiple antipsychotic medications were lowering his sodium levels. These problems were resolved once the medications were slowly withdrawn and discontinued. Sam was also on a number of other psychotropic medications of questionable value; these were rationalized. A preference and sensory assessment was completed by the occupational therapist, indicating that Sam showed a preference for certain foods and vibrating objects. A Functional Analysis (FA) was conducted by the behavior therapist; however, the results were inconclusive. An external consultation was requested from a psychologist with extensive experience in SIB; the psychologist recommended using a helmet with a face shield to allow Sam to move around freely without the possibility of biting himself.

Arm splints and bite resistant gloves were applied while Sam was sitting on the bed with his arms released from mechanical restraint. He was reinforced with preferred items for keeping the gloves on and not biting. However, Sam could not tolerate the splint and gloves and this intervention was discontinued. A helmet with a face guard and a custom made compression garment were tried next. The compression garment was made of scratch resistant material which would provide protection against scratching and biting as well as a mild pressure sensation. The helmet and compression garment were worn initially whenever 
Sam was released from restraints. The helmet was gradually faded to the point that Sam was not wearing it any more. He continued to wear the compression garment under regular clothing during the day, without an increase in SIB. At night, Sam was placed in mechanical restraints. The last part of the plan called for the fading of mechanical restraints until Sam was able to sleep and rest in bed without engaging in SIB. Unfortunately, Sam recently sustained an injury to his finger that required surgery. As a result, mechanical restraints are needed until his finger heals. Once this has been accomplished, staff will once again attempt to fade his restraints and prevent SIB from occurring.

\section{Scenario 2: Bill}

"Bill" is a 63-year-old man who has a diagnosis of mild-to-moderate intellectual disability and schizoaffective disorder. He lives in a group home that is run by a developmental services agency. Over a 30year period, Bill has received services from a dual diagnosis team and a schizophrenia program and has had admissions to general psychiatry units in local hospitals and a specialized mental health facility. Bill was recently admitted to the Geriatric Mental Health Inpatient Service that is also part of the Underserved Populations Program.

Until the age of 50 , Bill had been stable on several versions of a long-acting antipsychotic medication and a mood stabilizer medication. However, in recent years, Bill started to display a number of problem behaviors when any type of care was provided, including outbursts, verbal threats to harm his workers and other clients in his group home, property damage and wandering in the streets. In an effort to manage these behaviors, Bill was prescribed additional psychotropic medications including antidepressants and more recently two antipsychotics as a result of visits to various community physicians and several emergency departments. Over time, this has contributed to the development of medical problems and a more pronounced behavioral presentation. At the same time, Bill received support from a behavior therapist with a community developmental services agency and case management from an adult outpatient schizophrenia case worker. Bill also participated in an outpatient day program several days per week. Unfortunately, his behavior deteriorated which led to his admission to the Geriatric Mental Health Inpatient Service.

\section{Assessment and Treatment}

Bill's inter-professional clinical team consisted of the psychiatrist, advanced practice clinician (nurse), unit nurses, hospitalist, pharmacist, occupational therapist, recreational therapist, dietician and social worker from the Geriatric Mental Health Inpatient Service. Together, they developed an inter-professional plan of care which involved participation from Bill and was supported by Bill's substitute decision maker. The psychologist and behavior therapist from the Adult Neurodevelopmental Service joined the geriatric inter-professional team to provide additional input. Bill was taking 3 different classes of psychotropic medication (which included two antipsychotics) prior to his admission to the inpatient unit. Due to the many years of various types and quantities of psychotropic medication, Bill had developed impaired renal function and metabolic syndrome resulting in moderate obesity, hypertension and impaired insulin and blood glucose regulation. The psychiatrist from the geriatric mental health team has a specialty in recognizing the physiological and psychological effects of antipsychotic polypharmacy on aging client populations. The approach taken by the psychiatrist was to apply best practice principles to realign pharmacotherapy due to the lack of evidence for the efficacy of antipsychotic polypharmacy [15]. The hospitalist performed a physical exam and was concerned about the possibility of delirium. Bill was subsequently diagnosed with pneumonia and received appropriate medical treatment. The psychologist was able to establish that Bill did not have an acute cognitive deterioration. The unit nurses tended to assist Bill with self-care routines and to follow a behavioral schedule with rewards and consequences based on behavioral response.

The inpatient team worked to establish meaningful and purposeful daily activities for Bill. These activities involved walks on the hospital grounds and led to Bill's eventual integration into the surrounding community to visit public places such as cafes and food shops. With the behavioral plan in place, the recreational therapist and occupational therapist focused on other activities of daily living. Inter-professional staff including the social worker and the personal support worker provided skills-based training to improve Bill's ability to make healthier choices in regard to his eating habits, realizing that he was more successful when appropriate food choices and quantities were made clear to him. During his passes into the community, Bill was able to make independent choices that helped prepare him to return to his group home.

The geriatric inter-professional team implemented a behavioral plan with clear and immediate consequences for Bill's verbal threats and a 
predictable/structured family visit schedule to reduce his outbursts. Team members monitored Bill when he was on independent passes in the community and developed tools to help him find his way back to the inpatient unit when he became confused. The behavior therapist and social worker helped support Bill's discharge back to the community by working with his care team to ensure his plan was implemented in a consistent manner.

\section{DISCUSSION}

Inter-professional collaborative approaches to care are particularly well-suited to address the multi-faceted and often multi-systemic needs of complex individuals with intellectual disability and mental health problems. In this article, we have described changes to the model of care for our program for adults with intellectual disability and/or autism spectrum disorder with mental health/behavior problems. We also provided information about our efforts to promote interprofessional team development and collaboration as part of our change management strategy. We recognize this is a "work in progress" that will require ongoing effort, support and evaluation [16]. We chose case examples to illustrate the range of complex issues that inter-professional mental health teams are facing with increased frequency, such as responding to the needs of individuals with co-morbid medical concerns $[17,18]$ as well as those who are aging and/or have autism spectrum disorders [19-21].

Implementing a new model of service and making changes to team structure and process can be a daunting task. Overall, staff in the program appear to be approaching this challenge with a sense of enthusiasm and commitment. Changes to team culture are becoming more evident. For example, explicit language about inter-professional collaboration is becoming embedded into everyday clinical activities. However, traditional views regarding hierarchical medical models of care are often ingrained, especially in a hospital setting. We have observed anecdotally that it is proving more difficult to change attitudes of long-time staff than newer members of the team who don't have preconceived ideas about working relationships.

In relation to next steps, our goal is to evaluate the impact of our efforts to support inter-professional collaboration by repeating the survey of leadership and team members. We are developing action plans to address barriers to better collaboration that were identified in the surveys. In particular, conflict resolution, negotiation of shared roles and responsibilities and having sufficient time to engage in inter-professional discussion will be examined. We also plan to obtain feedback from our clients and their caregivers regarding their experiences receiving services from our inter-professional mental health team [22].

\section{REFERENCES}

[1] Summers J, Bradley E, Flannery J. Developmental disabilities and mental ill-health. In Brown I, Percy M, editors. Developmental disabilities in Ontario. $3^{\text {rd }}$ ed. Toronto, Ontario: Association on Developmental Disabilities 2011; p. 641-72.

[2] Reid J, Summers J, Adamson J, Habjan B, Meister C, Gignac, V. Mental health issues in clients with severe communication impairments. In Griffiths D, Stavrakaki C, Summers $\mathrm{J}$, editors. An introduction to the mental health needs of persons with developmental disabilities. Sudbury, Ontario: Habilitative Mental Health Resource Network 2002; p. 539-59.

[3] Bouras N, Szymanski L. Services for people with mental retardation and psychiatric disorders: US-UK comparative overview. Int J Social Psychiatry 1997; 43: 64-71. http://dx.doi.org/10.1177/002076409704300106

[4] Department of Developmental Disability Neuropsychiatry. Accessible mental health services for people with an intellectual disability: A guide for providers. University of New South Wales: Department of Developmental Disability Neuropsychiatry 2014.

[5] Radford JP. Towards a post-asylum society: A brief history of developmental disability policy in Ontario. In Brown I, Percy $M$, editors. Developmental Disabilities in Ontario. $3^{\text {rd }}$ ed. Toronto, Ontario: Association on Developmental Disabilities 2011; p. 25-40.

[6] Summers J, Bradley E, Fletcher R. (in press). People with intellectual and developmental disabilities and mental health needs. In Wehmeyer M, Brown I, Percy M, Shogren K, Fung $A$, editors. A comprehensive guide to intellectual and developmental disabilities. $2^{\text {nd }}$ ed. Baltimore: Paul $H$. Brookes.

[7] D'Amour D, Ferrada-Videla M, San Martin Rodriguez L, Beaulieu M. The conceptual basis for interprofessional collaboration: Core concepts and theoretical frameworks. J Interprof Care 2005; 19: 116-31.

http://dx.doi.org/10.1080/13561820500082529

[8] Health Force Ontario. Implementing interprofessional care in Ontario: Final report of the Interprofessional Care Strategic Implementation Committee 2010.

[9] Rosen A, Callaly T. Interdisciplinary teamwork and leadership: Issues for psychiatrists. Australas Psychiatry 2005; 13(3): 234-40.

http://dx.doi.org/10.1111/j.1440-1665.2005.02195.x

[10] Summers J, Boyd K, Reid J, Adamson J, Habjan B, Gignac $\mathrm{V}$, et al. The interdisciplinary mental health team. In Griffiths D, Stavrakaki C, Summers J, editors. An introduction to the mental health needs of persons with developmental disabilities. Sudbury, Ontario: Habilitative Mental Health Resource Network 2002; p. 325-57.

[11] Canadian Interprofessional Health Collaborative. A national interprofessional competency framework. Vancouver: College of Health Disciplines 2010.

[12] Registered Nurses' Association of Ontario. Developing and sustaining interprofessional health care: Optimizing 
patients/clients, organizational and systems outcomes. Toronto, Canada: Registered Nurses' Association of Ontario 2013.

[13] Wood S, Gangadharan S, Tyrer F, Gumber R, Devapriam J, Hiremath $A$, et al. Successes and challenges in the implementation of care pathways in an intellectual disability service: Health professionals' experiences. J Policy Pract Intellect Disabil 2014; 11: 1-7. http://dx.doi.org/10.1111/jppi.12063

[14] Vinokur-Kaplan D. Enhancing the effectiveness of interdisciplinary mental health treatment teams. Adm Policy Ment Health 1995; 22: 521-30. http://dx.doi.org/10.1007/BF02106536

[15] Borlido C, Remington G, Graff-Guerrero A, Arenovich T, Hazra M, Wong A. et al. Switching from 2 antipsychotics to 1 antipsychotic in schizophrenia: A randomized, double-blind, placebo-controlled study. J Clin Psychiatry 2016; 77: e14e20.

http://dx.doi.org/10.4088/JCP.14m09321

[16] Slevin E, Truesdale-Kennnedy M, McConkey R, Barr O, Taggart L. Community learning disability teams: Developments, composition and good practice. J Intellect Disabil 2008; 12: 59-79. http://dx.doi.org/10.1177/1744629507083583
[17] Azimi K, Modi M, Hurlburt J, Lunsky Y. Occurrence of medical concerns in psychiatric outpatients with intellectual disabilities. J Mental Health Res Intellect Disabil 2016. http://dx.doi.org/10.1080/19315864.2015.1108377

[18] Charlot L, Abend S, Ravin P, Mastis K, Hunt A, Deutsch C. Non-psychiatric health problems among psychiatric inpatients with intellectual disabilities. J Intellect Disabil Res 2011; 55: 199-209, DOI: 10.1111/j.1365-2788.2010.1294.x

[19] Lunsky Y, Gracey C, Bradley E. Adults with autism spectrum disorders using psychiatric hospitals in Ontario: Clinical profile and service needs. Res Autism Spectr Disord 2009; 3 : 1006-13. http://dx.doi.org/10.1016/j.rasd.2009.06.005

[20] Mukaetova-Ladinska EB, Perry E, Baron M, Povey C. Ageing in people with autism spectrum disorder. Int J Geriatr Psychiatry 2012; 27: 109-18. http://dx.doi.org/10.1002/gps.2711

[21] Thorpe L. Mental health and the intellectually disabled elderly. Psychiatr Times 2005; 22: 88-90.

[22] Venville A, Sawyer AM, Long M, Edwards N, Hair S. Supporting people with an intellectual disability and mental health problems: A scoping review of what they say about service provision. J Mental Health Res Intellect Disabil 2015 8: 186-212, DOI: 10.1080/1935864.2015.1069912.

Received on 22-02-2016

\section{DOI: http://dx.doi.org/10.6000/2292-2598.2016.04.01.2}

(C) 2016 Summers et al.; Licensee Lifescience Global.

This is an open access article licensed under the terms of the Creative Commons Attribution Non-Commercial License (http://creativecommons.org/licenses/by-nc/3.0/) which permits unrestricted, non-commercial use, distribution and reproduction in any medium, provided the work is properly cited. 